\title{
A new hypothesis on the manifestation of cerebral malaria: The secret is in the liver ${ }^{\text {th }}$
}

\author{
Yuri Chaves Martins ${ }^{\mathrm{a}, \mathrm{b}, *}$, Cláudio Tadeu Daniel-Ribeiro ${ }^{\mathrm{a}}$ \\ ${ }^{a}$ Laboratório de Pesquisas em Malária, Instituto Oswaldo Cruz and Centro de Pesquisa Diagnóstico e Treinamento em Malária, Fiocruz, Rio de Janeiro, RJ, Brazil \\ ${ }^{\mathrm{b}}$ Department of Pathology, Albert Einstein College of Medicine, The Bronx, NY, USA
}

\section{A R T I C L E I N F O}

\section{Article history:}

Received 4 June 2013

Accepted 6 August 2013

\begin{abstract}
A B S T R A C T
Despite the abundance of information on cerebral malaria (CM), the pathogenesis of this disease is not completely understood. At present, two nonexclusive dominant hypotheses exist to explain how the neurological syndrome manifests: the sequestration (or mechanical) hypothesis and the inflammatory hypothesis. The sequestration hypothesis states that sequestration of Plasmodium falciparum-parasitized red blood cells (pRBCs) to brain capillary endothelia causes obstruction of capillary blood flow followed by brain tissue anoxia and coma. The inflammatory hypothesis postulates that $P$. falciparum infection releases toxic molecules in the circulation, inducing an imbalanced systemic inflammatory response that leads to coagulopathy, brain endothelial cell dysfunction, accumulation of leukocytes in the brain microcirculation, blood brain barrier (BBB) leakage, cerebral vasoconstriction, edema, and coma. However, both hypotheses, even when considered together, are not sufficient to fully explain the pathogenesis of CM. Here, we propose that the development of acute liver failure (ALF) together with BBB breakdown are the necessary and sufficient conditions for the genesis of CM. ALF is characterized by coagulopathy and hepatic encephalopathy (HE) in a patient without pre-existing liver disease. Signs of hepatic dysfunction have been shown to occur in $2.5-40 \%$ of CM patients. In addition, recent studies with murine models demonstrated that mice presenting experimental cerebral malaria (ECM) had hepatic damage and brain metabolic changes characteristic of HE. However, the occurrence of CM in patients with mild or without apparent hepatocellular liver damage and the presence of liver damage in non-CM murine models indicate that the development of ALF during malaria infection is not the single factor responsible for neuropathology. To solve this problem, we also propose that BBB breakdown contributes to the pathogenesis of $\mathrm{CM}$ and synergizes with hepatic failure to cause neurological signs and symptoms. BBB dysfunction would thus occur in $\mathrm{CM}$ by a mechanism similar to the one occurring in sepsis and is in agreement with the inflammatory hypothesis. Nevertheless, differently from in the inflammatory hypothesis, BBB leakage would facilitate the penetration of ammonia and other toxins into the brain parenchyma, but would not be sufficient to cause CM when occurring alone. We believe our hypothesis better explains the pathogenesis of CM, does not have problems to deal with the exception data not explained by the previous hypotheses, and reveals new targets for adjunctive therapy.
\end{abstract}

(c) 2013 Elsevier Ltd. All rights reserved.

\section{Introduction}

Cerebral malaria (CM) is a life-threatening complication of Plasmodium falciparum malaria that remains a major public health

\footnotetext{
Support: YCM is supported by the Inter-hemispheric Research/NIH Global Infectious Disease Research Training Program (D43 TW007129). CTDR is recipient of a Research Productivity Fellowship from the National Brazilian Council for Scientific and Technological Development (CNPq) and from the Foundation for Research Support in the State of Rio de Janeiro (Faperj) as a "Cientista do Nosso Estado".

* Corresponding author at: Department of Pathology, Albert Einstein College of Medicine, 1300 Morris Park Ave, Golding Building, Room 704, The Bronx, NY 10461, USA. Tel.: +1 718430 2522; fax: +1 7184308541 .

E-mail address: yuri.chaves@einstein.yu.edu (Y.C. Martins).
}

problem in Africa and South East Asia [1]. The World Health Organization defines $\mathrm{CM}$ as the presence of $P$. falciparum parasitemia in conjunction with a coma persisting for at least $1 \mathrm{~h}$ after termination of a seizure or correction of hypoglycemia and without the presence of other causes of encephalopathy [2]. CM mortality rate remains high (10-20\% in children) despite artemisinin-based antimalarial treatment $[3,4]$. In addition, the disease causes significant long-term neuro-cognitive deficits in $10-20 \%$ of survivors [5-7].

The pathogenesis of CM has been studied extensively over several decades [8,9]. Despite the abundance of information generated on the disease, its pathogenesis remains largely unclear. There are currently two nonexclusive dominant hypotheses to explain how CM manifests: the sequestration (or mechanical) hypothesis and the inflammatory hypothesis [8,10-12]. Although both hypotheses 
claim to explain, at least partially, the pathogenesis of $\mathrm{CM}$, no adjunctive therapy has emerged from either hypothesis to address the clinical management or prevention of disease [13].

The sequestration hypothesis is based on the phenomena of adhesion of red blood cells (RBCs) parasitized byP. falciparum trophozoites and schizonts to both brain capillary endothelia and uninfected RBCs leading to sequestration and rosette formation, respectively [11]. These events would result in obstruction of capillary blood flow, focal brain tissue anoxia and decreased removal of waste products, which is thought to lead to coma [9,14]. Sequestration results from the binding of $P$. falciparum erythrocyte membrane protein 1 (PfEMP-1), present on the membrane of parasitized RBCs (pRBCs), with endothelial cell receptors such as intercellular adhesion molecule 1 (ICAM-1) [15,16]. Parasitized and uninfected RBCs also become less deformable during $P$. falciparum infection [14] which, together with rosetting, leads to mechanical plugging of capillaries. Studies showing that patients infected with P. falciparum clones prone to brain sequestration are more susceptible to $\mathrm{CM}$ development than patients infected with non-neurotropic clones corroborate this hypothesis $[17,18]$. However, the sequestration hypothesis does not explain some facts: (a) the absent or low correlation between parasitemia and mortality [19,20]; (b) the low rates of neurological deficits after recovery from coma in $\mathrm{CM}$ patients when compared to other neurological pathologies that cause brain anoxia [21,22]; (c) the increasing number of case reports of CM by infection with Plasmodium vivax, which is generally believed to neither sequester nor decrease RBC deformability [2326]; (d) the potential for P. falciparum to adhere to the microvasculature of other organs such as the heart and the small intestine without causing significant pathology [11].

The inflammatory hypothesis postulates that $P$. falciparum $\mathrm{pRBC}$ lysis releases both parasite-derived toxins and host intracellular molecules in the circulation inducing a systemic inflammatory response that leads to coagulopathy, brain endothelial cell dysfunction, accumulation of leukocytes in the brain microcirculation, blood brain barrier (BBB) leakage, cerebral vasoconstriction and edema $[10,12,13,27,28]$. These pathological alterations activate microglia and damage astrocytes and neurons, leading to coma. Parasite toxins (glycosylphosphatidylinositol [GPI], hemozoin) and intracellular molecules (heme) contain pathogen-associated molecular patterns (PAMPs) that are recognized by pattern recognition receptors present in cells of the innate immune system such as monocytes and neutrophils as well as on endothelial cells [29$31]$. Activation of these cells induces the secretion of pro-inflammatory cytokines like TNF- $\alpha$, IFN- $\gamma$, and LT- $\alpha$. Activated innate immune cells together with pro-inflammatory cytokines act by recruiting $\mathrm{CD}^{+}$and $\mathrm{CD}^{+} \mathrm{T}$ cells, which exacerbates the process by leading to the production of more pro-inflammatory cytokines $[12,30]$. This inflammatory response is beneficial at first, reducing parasite growth and activating catabolic pathways to eliminate parasite toxins and host molecules that can be dangerous when present in high amounts $[30,32]$. However, at later stages, the inflammatory response is not properly regulated and causes damage to the host $[31,32]$. High levels of hemoglobin released in the plasma following parasite replication in RBCs leads to the generation of free heme, a toxic metabolite which scavenges nitric oxide (NO), causing endothelial disturbances and damage to the BBB $[31,33,34]$. Activated $\mathrm{CD}^{+} \mathrm{T}$ cells also induce endothelial cell impairment by perforin-mediated mechanisms, contributing to blood-brain barrier (BBB) leakage and brain edema and leading to entry of cytokines and malaria antigens into the brain environment [35]. Endothelial activation also induces the overexpression of adhesion molecules (ICAM-1), allowing pRBC, platelet, and leukocyte adhesion to endothelia. Stimulation of endothelial cells by pro-inflammatory cytokines also increases the production of endothelin-1, a potent vasoconstrictor that, in the absence of NO, would cause brain ischemia [36-38]. In addition, activated monocytes activate intravascular coagulation components and platelets, leading to the consumption of clotting factors, which then predisposes to hemorrhages [12]. This pro-coagulant state also contributes to the formation of rosettes, which would be considered analogous to mini-thrombi [12]. Other mediators such as $\gamma \delta$ T cells, low levels of anti-inflammatory cytokines, microparticles and prostaglandins produced by the parasite seem to be involved in the pathogenesis by contributing to increased inflammation and endothelial cell activation [13,36,39-41]. The inflammatory hypothesis is mainly based and supported by human studies showing association between inflammatory mediators and development of $\mathrm{CM}$ and by experimental studies with mouse models of $\mathrm{CM}$ induced by Plasmodium berghei ANKA [13,39]. However, the inflammatory hypothesis also cannot fully explain the pathogenesis of $\mathrm{CM}$ for the following reasons: (a) high levels of inflammatory mediators are found during non-lethal $P$. vivax infection and in strains of mice resistant to experimental $\mathrm{CM}$ (ECM) development when infected by $P$. berghei ANKA (PbA) [12,42]; (b) anti-inflammatory agents do not improve and, in some instances, exacerbate the clinical course of disease in humans $[43,44]$; (c) BBB leakage is present in non-CM patients and in non-ECM murine models [45]; (d) there is not a clear definition of the sequence of events during the course of disease and the precise contribution of each process to adverse outcome in CM patients [13].

These data suggests that both hypotheses, even when considered together, are not sufficient to fully explain the pathogenesis of $\mathrm{CM}$. We hypothesize that the development of acute liver failure (ALF) together with BBB breakdown are the only necessary and sufficient conditions for the genesis of cerebral malaria. In the present article, we describe how this hypothesis could better explain the pathogenesis of CM.

\section{Hypothesis}

\section{ALF causes hepatic encephalopathy, a condition present in cerebral malaria}

ALF is defined by the presence of coagulopathy (International Normalized Ratio [INR] > 1.5) and hepatic encephalopathy (HE) in a patient without pre-existing liver disease [46,47]. Coagulopathy occurs mainly because of a decrease in the synthesis of clothing factors and thrombocytopenia during ALF [47]. HE is defined as any degree of altered mentation in a patient with ALF and can range from mild confusion to seizures and coma [47,48]. Although the precise mechanism of HE is not completely understood, studies indicate that loss of the liver detoxifying function causes hyperammonemia, which results in an accumulation of this toxic metabolite in the brain together with its detoxification product glutamine $[46,48]$. This disruption in brain ammonia metabolism and consequent increase in glutamine concentration inside astrocytes is believed to cause cerebral edema, increased intracranial pressure and possibly brain parenchyma herniation [48]. In addition, other molecules such as myo-inositol, phosphocholine and taurine are thought to be involved in the pathogenesis of $\mathrm{HE}$ [48]. Disease progression contributes to altered expression of glutamate and glutamine transporters. Therefore, hepatic dysfunction per se can cause increased propensity for hemorrhages, brain dysfunction and coma.

Hepatic dysfunction ranging from mild to fulminant has been described in $P$. falciparum and $P$. vivax malaria with an incidence between 2.5 and 40\% [49-52]. Different degrees of hepatic dysfunction and jaundice have also been shown to occur in CM patients and are associated with poor prognosis in some studies [53-55]. Although a relationship between liver damage and CM 
was not found in previous studies with adult patients [56,57], a strong association between increased hemozoin-laden Kupffer cells (a sign of liver inflammation) and CM was recently shown in Malawian children [53]. Cerebral edema, a hallmark of ALF, has also been observed in CM patients [4,58].

Data from human studies have been corroborated by findings in murine models of ECM. P. berghei ANKA infection causes liver damage in ECM-susceptible mice [59,60]. Hepatic damage is evident by the presence of activated, pigment-containing leukocytes adhering to the endothelium of sinusoids and large vessels of the liver, hypertrophic Kupffer cells saturated with malarial pigment, and moriform vacuolization of hepatocytes in liver histology of ECM mice [60]. In agreement with these findings, ECM mice show an increase in hepatic transaminases (AST and ALT) and modulation of the cytochrome P450 enzymatic activity during P. berghei ANKA infection $[59,61,62]$. More strikingly, studies using magnetic resonance spectroscopy show that there is a disturbance in brain metabolism in ECM mice characterized by increased glutamine and decreased myo-inositol and glycerophosphocholine, a pattern characteristic of hepatic encephalopathy [63-65]. ECM-susceptible mice also present brain edema and astrocyte swelling when infected with $P$. berghei ANKA, both hallmarks of ALF $[36,63,66]$.

Taken together, these data indicate that acute hepatic dysfunction occurs in ECM and strongly suggest its occurrence in CM patients. In addition, this hepatic dysfunction causes brain metabolic alterations characteristic of HE in ECM. Therefore, we consider that the occurrence of ALF in CM patients may be the cause of the development of neurological signs and coma.

\section{Mechanisms by which liver damage could occur in CM}

The mechanism by which malaria infection causes liver damage is not completely known. Reduction in portal venous flow as a consequence of micro-occlusion by $\mathrm{pRBC}$ and rosettes, intrahepatic cholestasis due to reticulo-endothelial blockage, hepatic microvilli dysfunction, apoptosis and oxidative stress due to an intense inflammatory response to the parasite are some possibilities [51]. In fact, one study showed a significant correlation between the amount of pRBC in the liver and the level of serum bilirubin and the enzyme aspartate transaminase (AST) in adults with severe malaria [57]. However, an association between pRBC sequestration in the liver or histologic evidence of hepatocellular damage and CM development was not found in children [53].

Studies that investigated the pathogenesis of liver damage during ECM showed that it is associated with infiltration of ${ }_{\gamma \delta} \mathrm{T}$ cells and $\mathrm{CD}^{+} \mathrm{T}$ cells that produce interferon- $\gamma$, IL-12 and IL-18 and is independent of $\mathrm{CD}^{+} \mathrm{T}$ cell accumulation [59,62]. Interestingly, the blockage of CTLA-4, a negative regulator of $\mathrm{T}$ cell function, exacerbates liver and brain pathology in ECM-susceptible mice and induces ECM in resistant mice $[62,67,68]$. Accordingly, interferon- $\gamma$, IL-12 and IL-18 are essential for the development of ECM $[67,69,70]$ and are associated with susceptibility to CM in humans $[32,71,72]$. In addition, studies with non-ECM models indicate that natural killer (NK) $\mathrm{T}$ cells, neutrophil activation, decreased levels of IL-27 and IL-22, and free heme overload due to hemolysis with posterior production of free radicals are involved in hepatic damage caused by murine malaria infection [73-76].

These data allow us to propose that the following mechanisms are involved in the pathogenesis of liver failure during $\mathrm{CM}$ : the release of pro-inflammatory mediators in the plasma (GPI, hemozoin, free heme) during hemolysis activates endothelial and Kupffer cells altering their normal anti-inflammatory/tolerogenic profile and creating a pro-inflammatory microenvironment in the liver. In response to activation, Kupffer cells release IL-12 and IL-18, thereby activating $\mathrm{CD}^{+}$and NK T cells $[73,77]$. Upon activation, $\mathrm{CD}^{+}$and NK T cells produce IFN- $\gamma$, TNF- $\alpha$ and other Th- 1 cytokines that further activate Kupffer cells in a positive feedback loop that increases the inflammatory response in the liver. Activated NK T cells also act by killing hepatocytes via perforin/granzyme cytolytic-mediated mechanisms [73]. In addition, the high amounts of free heme in the plasma, released after oxidation of free hemoglobin, activates liver endothelial cells that increase the expression of adhesion molecules such as ICAM-1, VCAM-1, CxCl-1 and CxCl-2 [74] and stimulates the migration of leukocytes to the organ. Free heme also accumulates in hepatocytes increasing the production of reactive oxygen species inside these cells which causes oxidative damage and induces apoptosis [74]. Further studies are needed to confirm whether all these mechanisms occur in ECM models and patients with $\mathrm{CM}$.

\section{ALF could explain the presence of coagulopathy and thrombocytopenia in $C M$}

Coagulopathy occurs in CM patients and ECM-susceptible mice $[12,51,78]$. Decreased levels of coagulation factors in serum together with consequential increased propensity for bleeding during thrombocytopenia correlates with the development of brain petechial hemorrhages in CM and ECM [12]. The mechanism behind the development of coagulopathy in CM patients is not completely understood. One hypothesis is that endothelial activation induced by the inflammatory response generated by the parasite causes a pro-coagulant state with posterior consumption of coagulation factors and activation of platelets during severe malaria [78]. The problems with this hypothesis are extensively discussed elsewhere [78], but the main criticism is that it is based on studies examining adult patients with severe malaria, not pediatric patients with $\mathrm{CM}$. On the other hand, the presence of coagulopathy and thrombocytopenia in CM patients could be explained, or at least potentiated, by a decrease in hepatic function. In fact, the liver synthesizes most of the proteins involved in coagulation and produces thrombopoietin, which regulates platelet production from megakaryocytes in the bone marrow [79]. Consequently, ALF patients have decreased levels of clotting factors, a tendency to bleed, and around $40 \%$ of them are thrombocytopenic [79]. Moreover, intravascular activation of coagulation also occurs in patients with ALF [79]. Therefore, we suggest that the presence of ALF contributes to the pathogenesis of coagulopathy and the characteristic petechial hemorrhages in the brain of $\mathrm{CM}$ patients.

\section{$B B B$ breakdown contributes to the development of encephalopathy during $C M$}

The occurrence of $\mathrm{CM}$ in patients with mild or without apparent hepatocellular liver damage $[53,56,57]$ and the presence of liver damage in non-CM murine models [56,57,73,74,76,77] indicate that the development of ALF during malaria infection is not the single factor responsible for neuropathology. One may, however, consider that BBB breakdown contributes to the pathogenesis of $\mathrm{CM}$ and synergizes with hepatic failure to induce neurological signs and symptoms. In this way, subjects presenting high levels of BBB dysfunction would need only mild hepatic damage to develop encephalopathy. In agreement, the opposite would also be true where subjects presenting low levels of BBB dysfunction would need severe hepatic damage to develop neurological signs.

BBB dysfunction occurs in CM and ECM [80]. The possible mechanisms causing BBB leakage in CM have been reviewed elsewhere [80-82]. Briefly, it is thought that adhesion of pRBC to the brain microvasculature causes brain endothelial cell activation with posterior inflammation. The local inflammation causes (or leads to) BBB leakage by decreasing the expression of tight junc- 
tions in the endothelia, causing apoptosis of endothelial cells, and activating $\mathrm{CD}^{+} \mathrm{T}$ cells which exert a direct cytotoxic effect against endothelial cells expressing parasite-derived antigens in an MHC class I context. This hypothesis, however, states that sequestration is triggering the process and, consequently, presents the same problems listed for the sequestration hypothesis.

We propose that BBB dysfunction occurs in CM by a mechanism similar to the one occurring in sepsis $[83,84]$ and is in agreement with the inflammatory hypothesis $[10,12,13,28]$. We hypothesize that CM pathophysiology involves an ischemic process, secondary to impairment of cerebral perfusion, not mainly derived from $\mathrm{pRBC}$ sequestration, and a neuro-inflammatory process that includes endothelial activation, alteration of the bloodbrain barrier and passage of neurotoxic mediators. In fact, data from studies in sepsis show that BBB dysfunction can be derived from a systemic inflammatory response [83]. Therefore, brain endothelial activation, which occurs because of the release of toxic molecules into plasma during hemolysis (GPI, hemozoin, free heme) would contribute to cerebral microcirculatory dysfunction, increasing the production of vasoconstrictive mediators and inhibiting molecules that induce vasodilation. This process would cause a state of global brain ischemia as shown in recent studies with ECM $[27,63,85-88]$. At the same time, endothelial activation would also trigger a local inflammatory response by increasing the expression of adhesion molecules, decreasing the expression of tight junctions, and producing pro-inflammatory cytokines. This would initiate the sequence of events described in the inflammatory hypothesis that would ultimately causes opening of the BBB. Contrarily to the proposed in the inflammatory hypothesis, we consider that BBB leakage alone would not lead to the development of $\mathrm{CM}$, but would, however, facilitate the diffusion and penetration of ammonia and other toxins derived from hepatic failure into the brain parenchyma leading to the development of neurological signs and symptoms.

In fact, under physiologic conditions, cerebral uptake of ammonia is mainly restricted to diffusion of the unprotonated form $\left(\mathrm{NH}_{3}\right)$; this is known as the diffusion hypothesis [89]. This occurs because the protonated form of ammonia $\left(\mathrm{NH}_{4}{ }^{+}\right)$does not easily cross membranes and the vast majority of brain endothelia do not present fenestrations. Opening of the BBB could, however, increase the diffusion of $\mathrm{NH} 4+$ into the brain parenchyma, thereby increasing its tissue concentration and triggering HE. This rationale could explain the presence of CM in patients with mild or without apparent hepatocellular liver damage, as mild hepatic damage could be enough to cause HE in the presence of BBB leakage. The same rationale could explain why, in some malaria murine models, liver damage occurs without a full-blown ECM picture. In these cases, BBB leakage would not occur or be too mild to cause coma. This should explain why ECM-susceptible mice infected with $P$. berghei NK65 do not develop neurological disease [59,73,77]. In this model, hepatic dysfunction occurs as well as low levels of BBB leakage $[73,77,90]$. Although $P$. berghei NK65-infected mice do not demonstrate full-blown ECM, they present brain metabolic disturbances characteristic of encephalopathy [91] and similar to the ones that occur in P. berghei ANKA-infected mice [64]. The difference between the two models could, therefore, be better explained by the degree of BBB dysfunction and it is not possible to consider $P$. berghei NK65-infected mice totally free of encephalopathy.

In addition, although still a matter of debate, ALF seems to cause disruption of the BBB [89,92]. It is possible to hypothesize, therefore that, if ALF is the cause of encephalopathy in $\mathrm{CM}$, it also contributes to the development of BBB leakage. It is important to emphasize, however, that BBB dysfunction secondary to ALF should be a secondary mechanism during malaria infection.

\section{Evaluation of the hypothesis}

It is not known if the dysfunction in ammonia metabolism characteristic of HE also occurs in the brain of CM patients. Although it is accepted that ECM models reproduce key characteristics of the disease in humans, one must be cautious to generalize conclusions based on findings in animal models [93,94]. Our hypothesis is mainly based on results from studies in ECM models, and human studies are needed to validate it.

In two studies, an association was not found between signs of liver damage and development of CM in adult patients in Thailand $[56,57]$. This could be explained by different degrees of BBB leakage in the study population. In both studies, $\mathrm{CM}$ and non-CM patients presented signs of liver damage in equal proportions and the authors concluded that liver damage is not associated with CM. However, CM patients may have also presented BBB leakage. As we postulated that BBB leakage facilitates the development of $\mathrm{CM}$, the presentation of neurological disease in the same population could vary by the degree of BBB leakage. The same rationale can be used to explain the presence of only mild signs of histologic hepatocellular damage in one study with Malawian children presenting CM [53].

There are studies showing that clones of $P$. vivax can adhere to brain endothelial molecules (ICAM-1) and form rosettes [95-97] These data could be used to explain the cases of $\mathrm{CM}$ caused by this specie of plasmodium. However, as far as we know, there are no studies definitively demonstrating an association between $P$. vivax adhesion in the brain microvasculature and development of $\mathrm{CM}$ [97].

\section{Predictions of the hypothesis}

\section{ALF associated with BBB leakage explains the vast majority of neuropathology which occurs in malaria}

Accepting that the presence of ALF and BBB leakage are the only conditions involved in the pathogenesis of $C M$ would address the uncertainties associated with the sequestration and inflammatory hypotheses.

The absent or low correlation between parasitemia and mortality $[19,20]$ can be explained by the fact that both liver and BBB dysfunction in the hypothesis are not only caused by direct parasitedamage to these organs, but also by the host immune response to molecules released in the circulation due to hemolysis. In fact, the immune response is subject to multiple genetic and environmental controls that can be better determinants of the outcome in CM than the levels of parasitemia.

The presence of low rates of neurological deficits after recovery from coma in CM patients $[21,22]$ could be attributed to the fact that ischemia is not the main determinant of neurologic dysfunction in our hypothesis. Ischemia, derived from vasoconstriction, contributes to BBB leakage, however neurological damage results from the accumulation of ammonia and other neurotoxins. In addition, although not a common feature, neurologic deficits have been shown to occur after recovery from ALF [46], similar to in CM.

Our hypothesis does not consider that pRBC sequestration to the brain microvasculature is necessary for the development of $\mathrm{CM}$. Such rational has the advantage of explaining the cases of $\mathrm{CM}$ caused by plasmodium species that does not have preference to adhere in the brain microvasculature such as $P$. vivax [23-26] and $P$. berghei ANKA. These species cause liver failure and systemic inflammation conditions that, when occurring together, would be enough to develop neurological damage. In addition, dissociation of sequestration from cerebral pathology would also explain why $P$. falciparum adheres to the microvasculature of other organs such 
as the heart and the small intestine without causing significant pathology [11].

Our hypothesis also addresses the issue of why anti-inflammatory agents do not improve or, in some instances, even exacerbate the clinical course of disease in CM $[43,44]$. In ALF, anti-inflammatory agents have proven effective as a prophylactic treatment for preventing liver damage, but there is no evidence that these agents are effective therapeutically for the treatment of established liver damage [46]. In accordance, anti-inflammatory interventions have been shown to prevent ECM, but few of them have been tested as adjunctive therapy in murine models [93]. N-acetylcysteine is a well-established treatment for paracetamol-induced ALF that has been tested in patients with severe malaria and showed no benefit [98]. However, N-acetylcysteine is a very specific treatment for paracetamol intoxication, and there is no evidence that it works for other forms of ALF [46].

Conditions that decrease hepatic function or predispose to $B B B$ dysfunction would predispose to CM development

A direct consequence from our hypothesis is that subjects with decreased hepatic function would be predisposed to the development of CM. In fact, a study with Vietnamese adults showed that CM patients had a greater risk of being positive for hepatitis B surface antigen relative to other manifestations of severe malaria [99]. In addition, co-infection with murine hepatitis virus induces ECM in P. berghei ANKA-infected BALB/c mice, a non-CM model (Martins Y.C., Carvalho L.J., Daniel-Ribeiro C.T., unpublished data). In agreement, the use of hepatotoxic drugs for the treatment of CM would be detrimental.

Using an analogous rationale, it is possible to predict that subjects predisposed to BBB dysfunction would be more susceptible to the development of $\mathrm{CM}$. This prediction is difficult to test in humans, but is easily testable in experimental models of BBB breakdown $[84,100]$. For example, mice deficient in tight junction proteins such as occludin or claudin $[101,102]$ would be more susceptible to the development of ECM when compared with wildtype mice.

\section{New possibilities for CM therapy}

Our hypothesis also opens an entire new field for discovery of adjunctive treatments for CM. Therapies to prevent or treat ALF would have great potential to improve survival in $\mathrm{CM}$ patients. Unfortunately, except for n-acetylcysteine, treatments for ALF and $\mathrm{HE}$ are only experimental $[46,48]$. Lactulose and rifaximin are effective therapies for HE in chronic liver failure, but do not seem to work in ALF $[46,48]$. The use of methionine sulfoximine to prevent the accumulation of glutamine in astrocytes demonstrated efficacy in both in vivo and in vitro models of ALF and could be easily tested in ECM models [48].

\section{Conflict of interest}

The authors declare no conflict of interest.

\section{Acknowledgments}

We thank Henry Joseph Shikani for his critical revision of the final manuscript. YCM is supported by the Inter-hemispheric Research/ NIH Global Infectious Disease Research Training Program (D43 TW007129). CTDR is recipient of a Research Productivity Fellowship from the National Brazilian Council for Scientific and Technological Development ( $\mathrm{CNPq}$ ) and from the Foundation for Research Support in the State of Rio de Janeiro (Faperj) as a "Cien- tista do Nosso Estado". Sponsors had no role in the collection, analysis and interpretation of data; in the writing of the manuscript; and in the decision to submit the manuscript for publication.

\section{References}

[1] WHO. World malaria report: 2012 In: W.H. Organization, editor. WHO Press, Geneva, 2012, p. 105.

[2] Severe falciparum malaria. World Health Organization communicable diseases cluster. Trans R Soc Trop Med Hyg 2000;94(Suppl. 1):S1-S90.

[3] Mwanga-Amumpaire J, Ndeezi G, Tumwine JK. Effect of vitamin A adjunct therapy for cerebral malaria in children admitted to Mulago hospital: a randomized controlled trial. Af Health Sci 2012;12:90-7.

[4] Mohanty S, Mishra SK, Patnaik R, et al. Brain swelling and mannitol therapy in adult cerebral malaria: a randomized trial. Clin Inf Dis 2011;53:349-55.

[5] Richardson ED, Varney NR, Roberts RJ, Springer JA, Wood PS. Long-term cognitive sequelae of cerebral malaria in Vietnam veterans. App Neuropsychol 1997;4:238-43.

[6] Boivin MJ, Bangirana P, Byarugaba J, et al. Cognitive impairment after cerebral malaria in children: a prospective study. Pediatrics 2007;119:e360-366.

[7] John CC, Bangirana P, Byarugaba J, et al. Cerebral malaria in children is associated with long-term cognitive impairment. Pediatrics 2008;122:e92-99.

[8] Martins YC, Carvalho LJ, Daniel-Ribeiro CT. Challenges in the determination of early predictors of cerebral malaria: lessons from the human disease and the experimental murine models. Neuroimmunomodulation 2009;16:134-45.

[9] Idro R, Jenkins NE, Newton CR. Pathogenesis, clinical features, and neurological outcome of cerebral malaria. Lancet Neurol 2005:4:827-40.

[10] Clark IA, Rockett KA. The cytokine theory of human cerebral malaria. Parasitol Today 1994;10:410-2.

[11] Berendt AR, Tumer GD, Newbold CI. Cerebral malaria: the sequestration hypothesis. Parasitol Today 1994;10:412-4.

[12] van der Heyde HC, Nolan J, Combes V, Gramaglia I, Grau GE. A unified hypothesis for the genesis of cerebral malaria: sequestration, inflammation and hemostasis leading to microcirculatory dysfunction. Trends Parasitol 2006;22:503-8.

[13] Higgins SJ, Kain KC, Liles WC. Immunopathogenesis of falciparum malaria: implications for adjunctive therapy in the management of severe and cerebral malaria. Expert Rev Anti-Infect Ther 2011:9:803-19.

[14] Dondorp AM, Kager PA, Vreeken J, White NJ. Abnormal blood flow and red blood cell deformability in severe malaria. Parasitol Today 2000;16:228-32.

[15] Springer AL, Smith LM, Mackay DO Nelson SO, Smith JD. Functional interdependence of the DBLbeta domain and c2 region for binding of the Plasmodium falciparum variant antigen to ICAM-1. Mol Biochem Parasitol 2004;137:55-64.

[16] Sherman IW, Crandall I, Smith H. Membrane proteins involved in the adherence of Plasmodium falciparum-infected erythrocytes to the endothelium. Biol Cell/under the auspices of the European Cell Biology Organization 1992:74:161-78.

[17] Claessens A, Adams Y, Ghumra A, et al. A subset of group A-like var genes encodes the malaria parasite ligands for binding to human brain endothelial cells. Proc Natl Acad Sci USA 2012;109:E1772-1781.

[18] Ochola LB, Siddondo BR, Ocholla $H$, et al. Specific receptor usage in Plasmodium falciparum cytoadherence is associated with disease outcome. PloS one 2011;6:e14741.

[19] Deloron P, Dumont N, Nyongabo T, et al. Immunologic and biochemical alterations in severe falciparum malaria: relation to neurological symptoms and outcome. Clin Inf Dis 1994;19:480-5.

[20] von Seidlein L, Olaosebikan R, Hendriksen IC, et al. Predicting the clinical outcome of severe falciparum malaria in african children: findings from a large randomized trial. Clin Inf Dis 2012;54:1080-90.

[21] Eling WM, Kremsner PG. Cytokines in malaria, pathology and protection. Biotherapy 1994:7:211-21.

[22] Clark IA, Cowden WB. The pathophysiology of falciparum malaria. Pharmacol Ther 2003;99:221-60.

[23] Thapa R, Patra V, Kundu R. Plasmodium vivax cerebral malaria. Indian Pediatr 2007;44:433-4.

[24] Rogerson SJ, Carter R. Severe vivax malaria: newly recognised or rediscovered. PLoS Med 2008;5:e136.

[25] Mahgoub H, Gasim GI, Musa IR, Adam I. Severe Plasmodium vivax malaria among Sudanese children at New Halfa Hospital, Eastern Sudan. Parasites Vectors 2012;5:154.

[26] Tanwar GS, Khatri PC, Sengar GS, et al. Clinical profiles of 13 children with Plasmodium vivax cerebral malaria. Ann Trop Paediatr 2011;31:351-6.

[27] Cabrales P, Zanini GM, Meays D, Frangos JA, Carvalho LJ. Murine cerebral malaria is associated with a vasospasm-like microcirculatory dysfunction, and survival upon rescue treatment is markedly increased by nimodipine. Am J Pathol 2010;176:1306-15.

[28] Clark IA, Alleva LM. Is human malarial coma caused, or merely deepened, by sequestration? Trends Parasitol 2009;25:314-8.

[29] Gazzinelli RT, Denkers EY. Protozoan encounters with Toll-like receptor signalling pathways: implications for host parasitism. Nat Rev Immunol 2006;6:895-906. 
[30] Nebl T, De Veer MJ, Schofield L. Stimulation of innate immune responses by malarial glycosylphosphatidylinositol via pattern recognition receptors. Parasitology 2005;130(Suppl.):S45-62.

[31] Ferreira A, Balla J, Jeney V, Balla G, Soares MP. A central role for free heme in the pathogenesis of severe malaria: the missing link? J Mol Med 2008;86:1097-111.

[32] Hunt NH, Golenser J, Chan-Ling T, et al. Immunopathogenesis of cerebral malaria. Int J Parasitol 2006;36:569-82.

[33] Gramaglia I, Sobolewski P, Meays D, et al. Low nitric oxide bioavailability contributes to the genesis of experimental cerebral malaria. Nat Med 2006;12:1417-22.

[34] Pamplona A, Ferreira A, Balla J, et al. Heme oxygenase-1 and carbon monoxide suppress the pathogenesis of experimental cerebral malaria. Nat Med 2007;13:703-10.

[35] Nitcheu J, Bonduelle O, Combadiere C, et al. Perforin-dependent braininfiltrating cytotoxic CD8+ T lymphocytes mediate experimental cerebral malaria pathogenesis. J Immunol 2003;170:2221-8.

[36] Machado FS, Desruisseaux MS, Nagajyothi, et al. Endothelin in a murine model of cerebral malaria. Expl Biol Med (Maywood, N.J.) 2006;231:1176-81.

[37] Dai M, Freeman B, Bruno FP, et al. The novel ETA receptor antagonist HJP-272 prevents cerebral microvascular hemorrhage in cerebral malaria and synergistically improves survival in combination with an artemisinin derivative. Life Sci 2012;91:687-92.

[38] Wenisch C, Wenisch H, Wilairatana P, et al. Big endothelin in patients with complicated Plasmodium falciparum malaria. J Infect Dis 1996;173:1281-4.

[39] de Souza JB, Riley EM. Cerebral malaria: the contribution of studies in animal models to our understanding of immunopathogenesis. Microbes Infect/ Institut Pasteur 2002;4:291-300.

[40] Coltel N, Combes V, Wassmer SC, Chimini G, Grau GE. Cell vesiculation and immunopathology: implications in cerebral malaria. Microbes Infect/Institut Pasteur 2006;8:2305-16.

[41] Haque A, Echchannaoui H, Seguin R, Schwartzman J, Kasper LH, Haque S. Cerebral malaria in mice: interleukin-2 treatment induces accumulation of gammadelta $\mathrm{T}$ cells in the brain and alters resistant mice to susceptible-like phenotype. Am J Pathol 2001;158:163-72.

[42] Hemmer CJ, Holst FG, Kern P, Chiwakata CB, Dietrich M, Reisinger EC. Stronger host response per parasitized erythrocyte in Plasmodium vivax or ovale than in Plasmodium falciparum malaria. Trop Med Int Health 2006;11:817-23.

[43] van Hensbroek MB, Palmer A, Onyiorah E, et al. The effect of a monoclonal antibody to tumor necrosis factor on survival from childhood cerebral malaria. J Infect Dis 1996;174:1091-7.

[44] Prasad K, Garner P. Steroids for treating cerebral malaria. Cochrane Database Syst Rev (Online) 2000;2:CD000972.

[45] Medana IM, Day NP, Sachanonta N, et al. Coma in fatal adult human malaria is not caused by cerebral oedema. Malaria J 2011;10:267.

[46] Lee WM. Recent developments in acute liver failure. Best Pract Res. Clin Gastroenterol 2012;26:3-16.

[47] Shawcross DL, Wendon JA. The neurological manifestations of acute liver failure. Neurochem Int 2012;60:662-71.

[48] Desjardins P, Du T, Jiang W, Peng L, Butterworth RF. Pathogenesis of hepatic encephalopathy and brain edema in acute liver failure: role of glutamine redefined. Neurochem Int 2012;60:690-6.

[49] Kochar DK, Tanwar GS, Khatri PC, et al. Clinical features of children hospitalized with malaria-a study from Bikaner, northwest India. Am J Trop Med Hyg 2010;83:981-9.

[50] Mohanty N, Satpathy SK, Nanda P. Hepatopathy in complicated falciparum malaria: report from eastern India. Trans R Soc Trop Med Hyg 2004;98:753-4.

[51] Autino B, Corbett Y, Castelli F, Taramelli D. Pathogenesis of malaria in tissues and blood. Mediterr J Hematol Infect Dis 2012;4:e2012061.

[52] Anand AC, Puri P. Jaundice in malaria. J Gastroenterol Hepatol 2005;20:1322-32.

[53] Whitten R, Milner Jr DA, Yeh MM, Kamiza S, Molyneux ME, Taylor TE. Liver pathology in Malawian children with fatal encephalopathy. Hum Pathol 2011;42:1230-9.

[54] Waller D, Krishna S, Crawley J, et al. Clinical features and outcome of severe malaria in Gambian children. Clin Inf Dis 1995;21:577-87.

[55] Wilairatana P, Looareesuwan S, Charoenlarp P. Liver profile changes and complications in jaundiced patients with falciparum malaria. Trop Med Parasitol 1994;45:298-302.

[56] Nacher M, Treeprasertsuk S, Singhasivanon P, et al. Association of hepatomegaly and jaundice with acute renal failure but not with cerebral malaria in severe falciparum malaria in Thailand. Am J Trop Med Hyg 2001;65:828-33.

[57] Prommano O, Chaisri U, Turner GD, et al. A quantitative ultrastructural study of the liver and the spleen in fatal falciparum malaria. Southeast Asian J Trop Med Public Health 2005;36:1359-70.

[58] Medana IM, Turner GD. Human cerebral malaria and the blood-brain barrier. Int J Parasitol 2006;36:555-68.

[59] Haque A, Best SE, Amante FH, et al. High parasite burdens cause liver damage in mice following Plasmodium berghei ANKA infection independently of CD8(+) T cell-mediated immune pathology. Infect Immun 2011:79:1882-8.

[60] Martins YC, Smith MJ, Pelajo-Machado M, et al. Characterization of cerebral malaria in the outbred Swiss Webster mouse infected by Plasmodium berghei ANKA. Int J Exp Pathol 2009;90:119-30.
[61] De-Oliveira AC, Da-Matta AC, Paumgartten FJ. Plasmodium berghei (ANKA): infection induces CYP2A5 and 2E1 while depressing other CYP isoforms in the mouse liver. Exp Parasitol 2006;113:256-61.

[62] Jacobs T, Plate T, Gaworski I, Fleischer B. CTLA-4-dependent mechanisms prevent $\mathrm{T}$ cell induced-liver pathology during the erythrocyte stage of Plasmodium berghei malaria. Eur J Immunol 2004;34:972-80.

[63] Penet MF, Viola A, Confort-Gouny S, et al. Imaging experimental cerebral malaria in vivo: significant role of ischemic brain edema. J Neurosci 2005;25:7352-8.

[64] Ghosh S, Sengupta A, Sharma S, Sonawat HM. Metabolic fingerprints of serum, brain, and liver are distinct for mice with cerebral and noncerebral malaria: a (1)H NMR spectroscopy-based metabonomic study. J Proteome Res 2012;11:4992-5004.

[65] Sanni LA, Rae C, Maitland A, Stocker R, Hunt NH. Is ischemia involved in the pathogenesis of murine cerebral malaria? Am J Pathol 2001;159:1105-12.

[66] Promeneur D, Lunde LK, Amiry-Moghaddam M, Agre P. Protective role of brain water channel AQP4 in murine cerebral malaria. Proc Natl Acad Sci USA 2013;110:1035-40.

[67] Hafalla JC, Claser C, Couper KN, et al. The CTLA-4 and PD-1/PD-L1 inhibitory pathways independently regulate host resistance to Plasmodium-induced acute immune pathology. PLoS Pathog 2012;8:e1002504.

[68] Jacobs T, Graefe SE, Niknafs S, Gaworski I, Fleischer B. Murine malaria is exacerbated by CTLA-4 blockade. J Immunol 2002;169:2323-9.

[69] Fauconnier M, Palomo J, Bourigault ML, et al. IL-12Rbeta2 is essential for the development of experimental cerebral malaria. J Immunol 2012;188:1905-14.

[70] Villegas-Mendez A, de Souza JB, Murungi L, et al. Heterogeneous and tissuespecific regulation of effector $\mathrm{T}$ cell responses by IFN-gamma during Plasmodium berghei ANKA infection. J Immunol 2011;187:2885-97.

[71] Marquet S, Doumbo O, Cabantous S, et al. A functional promoter variant in IL12B predisposes to cerebral malaria. Hum Mol Genet 2008;17:2190-5.

[72] Nagamine Y, Hayano M, Kashiwamura S, et al. Involvement of interleukin-18 in severe Plasmodium falciparum malaria. Trans R Soc Trop Med Hyg 2003;97:236-41.

[73] Adachi K, Tsutsui H, Seki E, et al. Contribution of CD1d-unrestricted hepatic DX5+ NKT cells to liver injury in Plasmodium berghei-parasitized erythrocyteinjected mice. Int Immunol 2004;16:787-98.

[74] Dey S, Bindu S, Goyal M, et al. Impact of intravascular hemolysis in malaria on liver dysfunction: involvement of hepatic free heme overload, NF-kappaB activation, and neutrophil infiltration. J Biol Chem 2012;287:26630-46.

[75] Mastelic B, do Rosario AP, Veldhoen M, et al. IL-22 protects against liver pathology and lethality of an experimental blood-stage malaria infection. Front Immunol 2012;3:85.

[76] Findlay EG, Greig R, Stumhofer JS, et al. Essential role for IL-27 receptor signaling in prevention of Th1-mediated immunopathology during malaria infection. J Immunol 2010;185:2482-92.

[77] Adachi K, Tsutsui H, Kashiwamura S, et al. Plasmodium berghei infection in mice induces liver injury by an IL-12- and toll-like receptor/myeloid differentiation factor 88-dependent mechanism. J Immunol 2001;167:5928-34.

[78] Moxon CA, Heyderman RS, Wassmer SC. Dysregulation of coagulation in cerebral malaria. Mol Biochem Parasitol 2009;166:99-108.

[79] Lisman T, Porte RJ. Activation and regulation of hemostasis in acute liver failure and acute pancreatitis. Semin Thromb Hemost 2010;36:437-43.

[80] Pino P, Taoufiq Z, Nitcheu J, Vouldoukis I, Mazier D. Blood-brain barrier breakdown during cerebral malaria: suicide or murder? Thromb Haemost 2005;94:336-40.

[81] Adams S, Brown H, Turner G. Breaking down the blood-brain barrier: signaling a path to cerebral malaria? Trends Parasitol 2002;18:360-6.

[82] Gay F, Zougbede S, N'Dilimabaka N, Rebollo A, Mazier D, Moreno A. Cerebral malaria: what is known and what is on research. Revue neurologique 2012;168:239-56.

[83] Adam N, Kandelman S, Mantz J, Chretien F, Sharshar T. Sepsis-induced brain dysfunction. Expert Rev Anti-Inf Ther 2013;11:211-21.

[84] Huber JD, Egleton RD, Davis TP. Molecular physiology and pathophysiology of tight junctions in the blood-brain barrier. Trends Neurosci 2001;24:719-25.

[85] Bertinaria M, Guglielmo S, Rolando B, et al. Amodiaquine analogues containing NO-donor substructures: synthesis and their preliminary evaluation as potential tools in the treatment of cerebral malaria. Eur J Med Chem 2011;46:1757-67.

[86] Martins YC, Clemmer L, Orjuela-Sanchez P, et al. Slow and continuous delivery of a low dose of nimodipine improves survival and electrocardiogram parameters in rescue therapy of mice with experimental cerebral malaria. Malaria J 2013;12:138.

[87] Martins YC, Zanini GM, Frangos JA, Carvalho LJ. Efficacy of different nitric oxide-based strategies in preventing experimental cerebral malaria by Plasmodium berghei ANKA. PloS one 2012;7:e32048.

[88] Zanini GM, Martins YC, Cabrales P, Frangos JA, Carvalho LJ. Snitrosoglutathione prevents experimental cerebral malaria. J Neuroimmune Pharmacol 2012;7:477-87.

[89] Sorensen M. Update on cerebral uptake of blood ammonia. Metab Brain Dis 2013;28:155-9.

[90] Lacerda-Queiroz N, Lima OC, Carneiro CM, et al. Plasmodium berghei NK65 induces cerebral leukocyte recruitment in vivo: an intravital microscopic study. Acta Tropica 2011;120:31-9. 
[91] Penet MF, Kober F, Confort-Gouny S, et al. Magnetic resonance spectroscopy reveals an impaired brain metabolic profile in mice resistant to cerebral malaria infected with Plasmodium berghei ANKA. J Biol Chem 2007;282:14505-14.

[92] Ott P, Larsen FS. Blood-brain barrier permeability to ammonia in liver failure: a critical reappraisal. Neurochem Int 2004;44:185-98.

[93] Carvalho LJ. Murine cerebral malaria: how far from human cerebral malaria? Trends Parasitol 2010;26:271-2.

[94] White NJ, Turner GD, Medana IM, Dondorp AM, Day NP. The murine cerebral malaria phenomenon. Trends Parasitol 2010;26:11-5.

[95] Marin-Menendez A, Bardaji A, Martinez-Espinosa FE, et al. Rosetting in Plasmodium vivax: a cytoadhesion phenotype associated with anaemia. PLoS Negl Trop Dis 2013;7:e2155.

[96] Carvalho BO, Lopes SC, Nogueira PA, et al. On the cytoadhesion of Plasmodium vivax-infected erythrocytes. J Infect Dis 2010;202:638-47.

[97] Costa FT, Lopes SC, Albrecht L, et al. On the pathogenesis of Plasmodium vivax malaria: perspectives from the Brazilian field. Int J Parasitol 2012;42:1099-105.
[98] Charunwatthana P, Abul Faiz M, Ruangveerayut R, et al. N-acetylcysteine as adjunctive treatment in severe malaria: a randomized, double-blinded placebo-controlled clinical trial. Crit Care Med 2009;37:516-22.

[99] Barcus MJ, Hien TT, White NJ, et al. Short report: hepatitis b infection and severe Plasmodium falciparum malaria in Vietnamese adults. Am J Trop Med Hyg 2002;66:140-2.

[100] Suidan GL, McDole JR, Chen Y, Pirko I, Johnson AJ. Induction of blood brain barrier tight junction protein alterations by CD8 $\mathrm{T}$ cells. PloS one 2008;3:e3037.

[101] Nitta T, Hata M, Gotoh S, et al. Size-selective loosening of the blood-brain barrier in claudin-5-deficient mice. J Cell Biol 2003;161:653-60.

[102] Saitou M, Furuse M, Sasaki H, et al. Complex phenotype of mice lacking occludin, a component of tight junction strands. Mol Biol Cell 2000;11:4131-42. 